\title{
Adapting group schema therapy for older adults with personality disorders: lessons learnt
}

\author{
A. C. Videler ${ }^{1,2 *}$ (iD, K. A. van Beest $^{3}$, M. A. Ouwens ${ }^{1,2}$, G. Rossi ${ }^{4}$, R. J. J. van Royen ${ }^{5}$ and \\ S. P. J. van Alphen ${ }^{1,3,4,6}$
}

${ }^{1}$ Clinical Centre of Excellence for Personality Disorders and Autism Spectrum Disorders in Older Adults, PersonaCura, GGz Breburg, Tilburg, The Netherlands, ${ }^{2}$ Tranzo, Scientific Centre for Care and Wellbeing of the Tilburg School of Social and Behavioral Sciences of Tilburg University, The Netherlands, ${ }^{3}$ Clinical Centre of Excellence for Personality Disorders in Older Adults, Mondriaan Hospital, Heerlen-Maastricht, The Netherlands, ${ }^{4}$ Vrije Universiteit Brussel (VUB), Department of Psychology (PE), Personality and Psychopathology research group (PEPS), Brussels, Belgium, ${ }^{5}$ Centre for Psychotherapy, Beuningen, The Netherlands and ${ }^{6}$ Tilburg University, Department of Medical and Clinical Psychology of the Tilburg School of Social and Behavioral Sciences of Tilburg University, The Netherlands

${ }^{\star}$ Corresponding author. Email: a.videler@ggzbreburg.nl

(Received 19 August 2021; revised 2 October 2021; accepted 4 October 2021)

\begin{abstract}
A first empirical study into group schema therapy in older adults with mood disorders and personality disorder (PD) features has shown that brief group schema therapy has potential to decrease psychological distress and to change early maladaptive schemas (EMS). Effect sizes however were smaller than those found in similar studies in younger adults. Therefore, we set out to adapt the treatment protocol for older adults in order to enhance its feasibility and outcome in this age group. We examined this adapted protocol in 29 older adults (mean age 66 years) with PDs from four Dutch mental health institutes. The primary outcome was symptomatic distress, measured by the Brief Symptom Inventory. Secondary outcomes were measured by the Young Schema Questionnaire, the Schema Mode Inventory, and the short version of the Severity Indices of Personality Problems. Contrary to our expectations, the adapted treatment protocol yielded only a small effect size in our primary outcome, and no significant improvement in EMS, modes and personality functioning. Patients pointed out that they were more aware of their dysfunctional patterns, but maybe they had not been able yet to work on behavioural change due to this schema therapy treatment being too brief. We recommend more intensive treatment for older patients with PDs, as they might benefit from more schema therapy sessions, similar to the treatment dosage in younger PD patients. They might also benefit from a combination of group therapy and individual treatment sessions.
\end{abstract}

\section{Key learning aims}

(1) How to adapt group schema therapy for older adults.

(2) How to explore feasibility and outcome.

(3) Treat older personality disorder patients as intensively as younger adults.

Keywords: acceptability; group therapy; older adults; schema therapy

\footnotetext{
(C) The Author(s), 2021. Published by Cambridge University Press on behalf of the British Association for Behavioural and Cognitive Psychotherapies. This is an Open Access article, distributed under the terms of the Creative Commons Attribution licence (https:// creativecommons.org/licenses/by/4.0/), which permits unrestricted re-use, distribution, and reproduction in any medium, provided the original work is properly cited.
} 


\section{Introduction}

Personality disorders (PDs) are complex mental health problems, associated with chronic dysfunction in several life domains across the life course (Hutsebaut et al., 2019; Newton-Howes et al., 2015). The essential feature of a PD is an enduring pattern of inner experience and behaviour that deviates markedly from the expectations of the individual's culture, causing significant distress in the patient and/or the environment (American Psychiatric Association, 2013). In older adults (over the age of 60), the behavioural manifestation and intensity of a PD differs due to biological, psychological and social aspects of ageing (van Alphen et al., 2015). Older adults with (comorbid) PDs in geriatric psychiatry are characterized by many symptoms, a complicated course of treatment and a poorer treatment prognosis compared with older people with other common mental disorders, such as anxiety disorders or depression (Newton-Howes et al., 2015). Moreover, prevalence of PDs in older adults is high, from 10.6 to $14.5 \%$ in community-dwelling older adults, between 30 and $50 \%$ in elderly out-patient populations, and up to $57.8 \%$ in nursing home residents (Penders et al., 2020). Notwithstanding, effectiveness studies on the treatment of PDs as the main focus of therapy in older adults are limited (Penders et al., 2020). The most important reason is that relatively little attention has been given to PDs in later life so far. Also, research in older patients is challenging because of the complex interaction between biological, social, cultural, and psychological factors.

Experts on treatment of PDs in older adults in Belgium and the Netherlands reached consensus that existing evidence-based therapies for PDs in younger adults up to the age of 50, such as schema therapy (ST), are also applicable to older adults over the age of 60 (van Alphen et al., 2012). This finding was cross-validated among US experts (Rosowsky et al., 2018).

ST was first described by Young (1990) to treat patients with pervasive, long-term psychological difficulties, such as PDs, who did not benefit enough from traditional cognitive behaviour therapy. ST is an integrative form of psychotherapy combining interventions from cognitive behavioural therapy, psychodynamic therapy, gestalt therapy, interpersonal therapy, and attachment theory in one unified treatment model. This model consists of three core concepts: early maladaptive schemas (EMS), schema coping styles, and schema modes. EMS are persistent and pervasive patterns of information processing, cognitions, emotions, memories, and attention preferences. These schemas originate early in our development through the interaction of aversive childhood experiences with biological and temperamental factors and are repeated and reinforced throughout life. Every child has basic needs for safety, love, acceptance, autonomy and adequate limit setting in order to undergo a healthy personality development (Young et al., 2003). When these basic needs are not fulfilled, EMS develop. In the ST model, EMS are considered the core elements of PDs (Young et al., 2003). Schema coping styles are developed in order to cope with the early environment that led to the development of EMS. Schema modes represent the combination of emotional and cognitive states and coping responses of an individual at a moment in time that are active together. Schema modes can be triggered by emotional events and may rapidly shift from one mode into another.

Schema therapy has been found effective for adult patients with borderline PD (Farrell et al., 2009; Giesen-Bloo et al., 2006; Nadort et al., 2009), and for adults with cluster C, paranoid, histrionic, and narcissistic PD (Bamelis et al., 2014). Schema therapy is promising for other complex mental disorders, including depression, post-traumatic stress disorder, eating disorders, and obsessive compulsive disorders (Cockram et al., 2010; Malogiannis et al., 2014; Renner et al., 2016; Simpson et al., 2010; Thiel et al., 2016).

Recently, two studies examined respectively the feasibility of ST in older adults, and the efficacy of ST in older adults with PDs. The first study investigated the feasibility of brief group schema therapy in older adults with chronic depression and comorbid PD features (Videler et al., 2014). 
Using a pre-mid-post design, a medium treatment effect was found on the reduction of symptomatic distress, EMS, and schema modes. Further analysis showed that the reduction in symptoms was mediated by a change in EMS, supporting not only the feasibility of ST in later life, but also the ability of ST to change EMS in older adults. Although the effect size on EMS change was significant $(d=0.38)$, it was smaller than the effect sizes found in two studies conducted in a group of younger adults and adolescents, respectively (van Vreeswijk et al., 2014: $d=0.75$; Renner et al., 2013: $d=0.88$ ). A possible explanation for this may be that EMS in younger adults and adolescents are more flexible and changeable during treatment compared with older adults (Videler et al., 2014). However, another and more likely explanation was that ST needs to be adapted to older adults in order to enhance its feasibility and effectiveness in this age group. Therefore, Videler et al. (2014) suggested integrating agespecific aspects into the treatment protocol in order to generate better treatment effects. Videler and colleagues (2014) also suggested several adaptations to the treatment protocol to meet the needs of older patients. Older adults probably need more time to learn the schema language and to recognize the triggering of schemas and modes in their personal life. Therefore, they may substantially benefit from having several individual ST sessions prior to the start of group therapy. Also, simplifying a number of cognitive techniques in the workbook, illustrated with examples that fit their experiential world, has been suggested to improve therapy outcome. Applying age-specific aspects in the treatment of PDs was also recommended in both aforementioned Delphi studies among experts in PDs in older adults in the Netherlands, Belgium and the US (Rosowsky et al., 2018; van Alphen et al., 2012).

The second study examined the effectiveness of 50 sessions of individual ST in eight older adults with cluster C PDs using a multiple-baseline case series design, and found considerable positive effects on PD pathology, symptomatic distress $(d=1.29)$, and quality of life $(d=0.63)$ (Videler et al., 2018).

Although these two studies provided support for the feasibility and the efficacy of ST in the treatment of older adults with PDs, further research is warranted to replicate these findings and more importantly, to enhance the treatment outcome of older adults with PDs by adapting ST to the needs of older people.

Based on these and other hypotheses in the literature on adapting ST for older adults (van Alphen et al., 2012; Videler et al., 2014; Videler et al., 2015; Videler et al., 2017), the following adjustments to the brief group schema therapy protocol were made: (a) adding two pre-therapy sessions and two additional group therapy sessions to give patients more time to learn the schema language and get acquainted with the cognitive techniques; (b) adapting the patient workbook by using examples that fit to the lived experience of older adults; (c) integrating experiential techniques like imagery and rescripting and chairwork; (d) compensating for the decline of social support by encouraging group cohesion, whereby the therapists act as a parent and the participants take the role of siblings, and also actively involving close relatives in the treatment; (e) adding treatment strategies on schema modes (e.g. group imagery, improvisation) and (f) contextualizing to a lifespan perspective by incorporating wisdom enhancement ('wisdom of their years') and reinforcing positive, or functional, schemas.

In this study we examined this adapted form of brief group schema therapy for older adults by replicating the study of Videler and colleagues (2014). Our hypothesis was that the adapted protocol would be feasible and associated with an enhanced effectiveness of group schema therapy in this age group compared with the findings of Videler and colleagues (2014). We expected to find a decline of symptomatic distress, EMS and mode severity, and more adaptive personality functioning, and a greater treatment effect in comparison with the earlier study by Videler et al. (2014). 


\section{Method}

Design

We conducted a multi-centre trial using an experimental pre-, mid- and post-test design in order to investigate the feasibility and explore the outcome of the adapted group schema therapy protocol for a group of personality-disordered older adults, with the exclusion of schizoid, schizotypal or anti-social PD.

\section{Participants}

Twenty-nine patients from four Dutch mental health institutes were included in this study: five patients from the Clinical Centre of Excellence for Personality Disorders in Older Adults, Mondriaan hospital, five patients from PersonaCura, Clinical Centre of Excellence for Personality Disorders and Autism in Older Adults, GGZ Breburg, six patients from the Centre for Old Age Psychiatry of GGZ Eindhoven, and 13 patients from the Old Age Psychiatry Department of GGZ Oost-Brabant.

Inclusion criteria were: (1) age 60 years old or older; (2) primary diagnosis of a PD according to the DSM-5 criteria, as assessed with the Dutch version of the Structured Clinical Interview for DSM-IV Axis II Personality Disorders (SCID II; First et al., 1997; Dutch translation by Weertman et al., 2000) supplemented with measurements on the Gerontological Personality Disorders Scale (GPS; van Alphen et al., 2006), and the Severity Indices of Personality Problems-Short Form (SIPP-SF; derived from the SIPP-118; Verheul et al., 2008); and (3) willingness to participate in the study. Exclusion criteria were: (1) severe cognitive impairments due to a neurodegenerative disease (MMSE <24); (2) a schizoid, schizotypal or anti-social PD; (3) substance abuse needing clinical detoxification; (4) lifetime prevalence of psychosis or bipolar disorder; (5) major psychotic depression; (6) learning disabilities (IQ < 75); or (7) patients with significant hearing or vision problems to such an extent that they could not participate in a group. All participants were informed about the study and signed an informed consent form.

\section{Procedure}

First, we adapted the brief group schema therapy protocol (Broersen and Van Vreeswijk, 2012) as described in the Introduction. In the two pre-therapy sessions, patients and their close relatives received information about ST and an individual schema-mode model was composed. Also, the first measurement of symptomatic distress, EMS and schema modes was administered. The second measurement was after 10 weeks of therapy and the last measurement was at the end of therapy. The results of these measurements were discussed with the patient and his or her close relative. The adapted treatment protocol consisted of 22 sessions, 20 weekly sessions of 90 minutes and two booster sessions of 90 minutes, one and two months after termination of treatment. Patients who missed a session were informed about the next session by one of the therapists. All therapists were certified ST therapists. Therapists were able to consult each other. At the start and every two months there was a supervision session with an experienced schema therapist, licensed as a ST supervisor by the Dutch Schema therapy association (fifth author). During the course of the study, no other treatment was allowed. Unless in case of clinical urgency, medication was kept constant.

\section{PD measures}

The Dutch version of the SCID-II (First et al., 1997; Weertman et al., 2000) was used for PD diagnosis according to DSM-5 Section II PDs. The SCID-II is a semi-structured interview that covers all 10 specific DSM-5 PDs as well as PD not otherwise specified (other specified PD in 
DSM-5). The interview contains 134 open-ended questions and begins with questions about behaviour and relationships of the patient. Thereafter, items assess the diagnostic criteria for each of the 10 standard PDs, organized one by one. Each PD criterion is rated as 1 (absent or false), 2 (subthreshold), or 3 (threshold or true). The SCID-II has shown good inter-rater reliability for the presence or absence of a PD in adults (Lobbestael et al., 2011).

The GPS (van Alphen et al., 2006) is a screening instrument to detect PDs in older adults. The GPS consists of a patient version and an informant version. Both versions consist of two scales: habitual behaviour (GPSHAB) and biographical information (GPSBIO). The GPSHAB scale assesses habitual behaviours that reflect the expression of a number of PD features. In the GPSBIO scale, important and recurrent events or decisions in life are linked to the presence or absence of DSM-5 PDs. The internal consistency (Cronbach's alpha) of the two scales ranges from poor (GPSHAB $\alpha=.57$ ) to acceptable (GPSBIO $\alpha=.77$; van Alphen et al., 2006). The test-re-test reliability of the GPSHAB and the GPSBIO subscales were moderate (Spearman's $r=.72$ ) and excellent (Spearman's $r=.89$ ), respectively. Sensitivity and specificity of the GPS patient version in an older psychiatric out-patient population was shown to be fair with sensitivity and specificity levels around 70\% (van Alphen et al., 2006). In this study, only the GPS patient version was used.

\section{Outcome measures}

The primary outcome variable was symptomatic distress, measured by the Brief Symptom Inventory (BSI; De Beurs, 2011; translated from Derogatis, 1975a). The BSI is a shorter version of the Symptom Checklist-90 (SCL-90; Derogatis, 1975b), and consists of 53 items. It is a self-report assessment instrument providing an overview of a patient's symptoms and their intensity using nine symptom scales. The reliability of the Dutch BSI scales is good and the convergent and divergent validity has been found to be satisfactory (De Beurs, 2011). The BSI is validated for older adults and, because it is less lengthy, also recommended for this age group (van Alphen et al., 2012).

As secondary outcome variable, the short version of the Severity Indices of Personality Problems (SIPP-SF, derived from the SIPP-118; Verheul et al., 2008) was used to assess limitations in personality functioning. The SIPP-SF is a self-report questionnaire that measures the core components of (mal)adaptive personality functioning, and provides indices of the severity of personality pathology. It consists of 60 items and measures five factors (i.e. Self-Control, Identity Integration, Relational Capacities, Responsibility, Social Concordance). There are four response categories: 1 = 'fully disagree', 2 = 'partly disagree', $3=$ 'partly agree', and $4=$ 'fully agree'. In a study of 150 community adults and 252 psychiatric outpatients, Ro and Clark (2009) reported acceptable alphas (i.e. .83-.89) across these subscales. The SIPP-SF was shown to have good construct validity to assess impaired personality functioning in older adults (Debast et al., 2018; Rossi et al., 2016). The internal reliability of the SIPP-SF domains was found to be good to excellent in a sample of older adult Dutch PD out-patients (Cronbach's alphas from $\alpha=.75$ to .91; van Reijswoud et al., 2021).

\section{Intervention-specific measures}

The Young Schema Questionnaire (YSQ; Young and Brown, 1994; Dutch translation by Sterk and Rijkeboer, 1997) was used to measure EMS. Research on the Dutch YSQ has provided support for its psychometric properties and clinical relevance (Rijkeboer et al., 2005; Rijkeboer and van den Bergh, 2006). The YSQ is a self-report assessment instrument of 205 items, to assess the 16 early maladaptive schemas as defined by Young et al. (2003): emotional deprivation, abandonment, mistrust/abuse, social isolation, defectiveness/shame, social undesirability, failure to achieve, dependence/incompetence, vulnerability to harm, enmeshment, subjugation, 
self-sacrifice, emotional inhibition, unrelenting standards, entitlement, and insufficient selfcontrol. Each of the items is rated using a 6-point Likert scoring format ranging from 'completely untrue' to 'describes me perfectly'. The YSQ is a relatively age-neutral instrument to assess EMS across the life course (Pauwels et al., 2014).

Schema modes were measured by the Schema Mode Inventory (SMI; Dutch translation Lobbestael et al., 2005). The SMI is a self-report assessment instrument of 118 items and measures 14 modes, divided into four types of modes: healthy modes, parent modes, child modes and coping modes. The Dutch SMI has excellent test-re-test reliability and the convergent and divergent validity of the subscales are satisfactory (Lobbestael et al., 2010).

\section{Statistical analyses}

The pre-, mid- and post-treatment measurements on the BSI, YSQ and SMI and the pre- and posttreatment measurement on the SIPP-SF were compared using paired samples $t$-tests. The strength of outcome was measured with Cohen's $d$ effect sizes for paired sample $t$-tests. Generally, $d<0.20$ is considered a small effect size, $d=0.50$ a medium effect size, and $d>0.80$ a large effect size.

Treatment success was determined by the classification of patients as recovered, improved, unchanged or deteriorated by Lambert et al. (2008). We first calculated changes between the BSI pre-treatment to post-treatment and assessed these changes for statistically reliable change. Next, it was determined whether patients who showed reliable change also passed the clinical cut-off point [reliable change scores were 18 for men and 19 for women, and clinical cut-off points 35 for men and 37 for women, based on norm group data provided in the Dutch manual of the BSI by De Beurs (2011)]. After these two steps each patient could be classified as recovered (reliable change and below the cut-off), improved (reliable change and above cut-off), unchanged (no reliable change) or deteriorated (reliable change in a negative direction). After calculating the residual change scores for the pre- to mid-, the mid- to end-, and pre- to end-of-treatment measurements on the BSI, YSQ and schema modes, hierarchical regression analysis was conducted to examine whether early process changes in EMS and schema modes predicted later outcome changes in symptoms, controlling for early outcome change scores and later process changes (Finkel, 1995). Inverse associations were also determined by regression analysis. To further analyse the data, we used the point-biserial correlation $(r)$ between a dichotomous independent variable and the scale scores on a dependent variable as a measure of effect size (Rosnow and Rosenthal, 1996). The pointbiserial correlation is a Pearson's product moment correlation with one variable dichotomous and one variable continuous and is mathematically related to Cohen's $d$ in the sense that $d=2 r / \sqrt{ }\left(1-r^{2}\right)$ under the assumption that one can legitimately assume that the group $n$-values are equal in each of the dichotomous variable groups. The following heuristic rules (Cohen, 1988) are available to interpret the effect size: $r=.10$ indicates a small effect, $r=.30$ indicates a medium effect, and $r=.50$ indicates a large effect. All the analyses were two-tailed with a significance level of $5 \%$, unless stated otherwise.

\section{Results}

\section{Attrition}

A total of 29 older out-patients were included in this study; three patients dropped out. One 58-year-old patient was included as she was referred for age-related problems, such as being widowed and coping with health issues. The mean age of the patients who completed treatment $(n=26)$ was 66 years (range $58-78)$. Of those who dropped out $(n=3)$, average age was 63 years (range 61-64). Table 1 shows an overview of the baseline characteristics of the patients who completed the treatment and those who dropped out. 
Table 1. Baseline characteristics

\begin{tabular}{|c|c|c|c|c|}
\hline & \multicolumn{2}{|c|}{ Treatment completers } & \multicolumn{2}{|c|}{ Drop-out patients } \\
\hline & Mean & Range & Mean & Range \\
\hline \multirow[t]{2}{*}{ Age (years) } & 66 & $58-78$ & 63 & $61-64$ \\
\hline & $n$ & Per cent & $n$ & Per cent \\
\hline Women & 21 & 81 & 2 & 67 \\
\hline Men & 5 & 19 & 1 & 33 \\
\hline \multicolumn{5}{|l|}{ Personality disorder } \\
\hline Obsessive compulsive personality disorder & 4 & 15 & 1 & 33 \\
\hline Dependent personality disorder & 3 & 12 & & \\
\hline Avoidant personality disorder & 3 & 12 & & \\
\hline Narcissistic personality disorder & 1 & 4 & & \\
\hline Borderline personality disorder & 6 & 23 & & \\
\hline Other specified personality disorder & 9 & 34 & 2 & 67 \\
\hline \multicolumn{5}{|l|}{ Co-morbid diagnoses } \\
\hline Mood disorder & 16 & 62 & 3 & 100 \\
\hline Anxiety disorder & 7 & 27 & & \\
\hline None & 3 & 11 & & \\
\hline
\end{tabular}

Table 2. Means, standard deviations, and effect sizes for the BSI, SIPP-SF, EMS and schema modes

\begin{tabular}{|c|c|c|c|c|c|c|c|}
\hline & \multicolumn{2}{|c|}{ Pre-treatment } & \multicolumn{2}{|c|}{ Mid-treatment } & \multicolumn{2}{|c|}{ Post-treatment } & \multirow[b]{2}{*}{ Effect sizes } \\
\hline & Mean & $S D$ & Mean & $S D$ & Mean & $S D$ & \\
\hline BSI & 74.29 & 39.06 & 70.60 & 33.01 & 67.12 & 31.62 & $.09 / .14 / .20$ \\
\hline YSQ total & 42.50 & 10.44 & 41.42 & 9.93 & 41.05 & 10.77 & $.11 / .04 / .14$ \\
\hline Functional modes & 49.58 & 13.75 & 47.38 & 15.61 & 50.94 & 13.43 & $.15 /-.24 /-.10$ \\
\hline Dysfunctional modes & 31.09 & 9.69 & 30.94 & 9.76 & 30.47 & 9.75 & $.02 / .05 / .07$ \\
\hline Child modes & 27.89 & 12.61 & 28.08 & 11.67 & 26.92 & 10.14 & $-.02 / .10 / .08$ \\
\hline Coping modes & 32.74 & 10.25 & 31.68 & 10.57 & 31.92 & 10.98 & $.10 /-.02 / .08$ \\
\hline Parent modes & 35.56 & 12.61 & 37.46 & 12.71 & 36.35 & 14.90 & $-.07 / .08 / .02$ \\
\hline SIPP-SF SC & 35.76 & 8.55 & n.a. & n.a. & 37.20 & 7.93 & n.a./ n.a./ -.17 \\
\hline SIPP-SF IdInt & 30.08 & 8.14 & n.a. & n.a. & 31.16 & 7.90 & n.a./ n.a./ -.14 \\
\hline SIPP-SF RC & 29.60 & 6.39 & n.a. & n.a. & 30.72 & 7.63 & n.a./ n.a./ -.16 \\
\hline SIPP-SF Res & 39.68 & 6.57 & n.a. & n.a. & 38.76 & 5.80 & n.a./ n.a./ .15 \\
\hline SIPP-SF SC & 37.24 & 6.63 & n.a. & n.a. & 37.48 & 7.17 & n.a./ n.a./ -.04 \\
\hline
\end{tabular}

BSI, Brief Symptom Inventory total score; YSQ, Young Schema Questionnaire. Functional schema modes are the healthy adult mode and happy child mode; dysfunctional schema modes are all the child modes, except for the happy child, coping modes and parent modes. SIPP-SF SC is the score on the factor Self-Control, SIPP-SF IdInt is the score on the factor Identity Integration, SIPP-SF RC on the factor Relational Capacities, SIPP-SF RES on the factor Responsibility and SIPP-SF SC on the factor Social Concordance on the short version of the Severity Indices of Personality Problems. Effect sizes (with paired sample $t$-tests) are based on the difference in scores from pretreatment to mid-treatment $(x /)$, from mid-treatment to end-of-treatment $(/ x /)$, and from pre-treatment to end-of-treatment $(/ / x)$ divided by the mean of the corresponding standard deviation. n.a., not available. Effect sizes were measured with Cohen's $d$ for paired sample $t$-tests.

\section{Treatment outcomes}

The results of the primary outcome analyses and the intervention-specific measurements are summarized in Table 2. None of the paired-sample $t$-tests was significant. The effect size of symptomatic distress from pre-treatment to end-of-treatment points in the direction of a small effect $(d=.20)$.

To determine treatment success, the classification of patients as recovered, improved, unchanged or deteriorated by Lambert et al. (2008) was used. Of all patients, $4 \%$ recovered, $12 \%$ improved, $73 \%$ remained unchanged and $12 \%$ deteriorated.

\section{Zero-order correlations}

Table 3 shows the zero-order correlations among the residual change scores on the BSI, YSQ total score and schema modes scores. The autocorrelations were not significant, indicating that changes 
Table 3. Zero-order correlations of residual change score

\begin{tabular}{|c|c|c|c|c|c|c|c|c|c|}
\hline & & 1 & 2 & 3 & 4 & 5 & 6 & 7 & 8 \\
\hline (1) & $\begin{array}{l}\text { Pre-treatment to mid-treatment } \\
\text { BSI }\end{array}$ & - & & & & & & & \\
\hline (2) & $\begin{array}{l}\text { Mid-treatment to end-treatment } \\
\text { BSI }\end{array}$ & -.068 & - & & & & & & \\
\hline (3) & $\begin{array}{l}\text { Pre-treatment to mid-treatment } \\
\text { YSQ }\end{array}$ & $.419^{\star}$ & .095 & - & & & & & \\
\hline (4) & $\begin{array}{l}\text { Mid-treatment to end-treatment } \\
\text { YSQ }\end{array}$ & $.454^{\star}$ & .331 & -.26 & - & & & & \\
\hline (5) & $\begin{array}{l}\text { Pre-treatment to mid-treatment } \\
\text { functional schema modes }\end{array}$ & -.326 & -.229 & $-.524^{\star \star}$ & -.108 & - & & & \\
\hline (6) & $\begin{array}{l}\text { Mid-treatment to end-treatment } \\
\text { functional schema modes }\end{array}$ & -.089 & $-.534^{\star \star}$ & -.116 & $-.402^{\star}$ & -.193 & - & & \\
\hline (7) & $\begin{array}{l}\text { Pre-treatment to mid-treatment } \\
\text { dysfunctional schema modes }\end{array}$ & $.478^{\star}$ & -.169 & $.556^{\star \star}$ & .076 & -.227 & -.018 & - & \\
\hline (8) & $\begin{array}{l}\text { Mid-treatment to end-treatment } \\
\text { dysfunctional schema modes }\end{array}$ & .299 & $.493^{\star}$ & -.077 & $.778^{\star \star}$ & -.203 & -.347 & -.133 & - \\
\hline
\end{tabular}

${ }^{*}$ Correlation is significant at the 0.05 level (two-tailed); ${ }^{*}$ correlation is significant at 0.01 level (two-tailed).

in the same variables at the beginning of the treatment were unrelated to changes in the same variables later in the treatment. Synchronous correlations for change scores showed moderate significant relations for most of the variables, indicating that change in process and outcome factors in the same period were modestly related. Cross-lagged correlations for change showed no significant relationships between pre- to mid-treatment change scores on YSQ total score and schema modes scores and mid- to end-of-treatment BSI change. The converse correlations were also not significant, except for the correlation between mid- to end-oftreatment YSQ change scores and the pre- to mid-treatment BSI change score.

\section{Mediation effects: testing cross-lagged associations with hierarchical regression analysis}

After calculating the residual change scores for the pre- to mid-, the mid- to end- and pre- to end-of-treatment measurements on the BSI, YSQ and schema modes, hierarchical regressions were used to examine whether early process changes in EMS and schema modes predicted later outcome changes in symptoms, controlling for early outcome change scores and later process changes (Finkel, 1995).

As shown in Table 4, despite the non-significant cross-lagged correlation, the pre- to midtreatment functional schema modes change score was a significant predictor of the variance in mid- to end-of-treatment BSI change score, after controlling for the other change scores. The correlation was negative, indicating that the decline in functional schema modes led to an increase of symptomatic distress. An additional 15\% of variance was accounted for, apart from the variance due to pre- to mid-treatment changes on BSI and for mid- to end-oftreatment changes scores on the functional schema modes. This assumes that the decline in functional schema modes led to an increase of symptomatic distress. The converse lagged association was not significant. Neither the pre- to mid-treatment YSQ change score, nor the pre- to mid-treatment dysfunctional modes score were significant predictors of the later change in symptomatic distress.

The converse lagged association of the pre- to mid-treatment BSI change score was significantly related to the later changes in EMS and dysfunctional schema modes. This implies that the magnitude of change in symptomatic distress at the beginning of the treatment predicts the eventual change in EMS and dysfunctional schema modes. 
Table 4. Summary of hierarchical regression analyses

\begin{tabular}{|c|c|c|c|c|}
\hline Variable & $\beta$ & SE $\beta$ & $R^{2}$ & $\Delta R^{2}$ \\
\hline \multicolumn{5}{|l|}{ Mid-to end-of-treatment BSI } \\
\hline Pre-treatment to mid-treatment BSI & -.439 & -.207 & & \\
\hline $\begin{array}{l}\text { Mid-treatment to end-of-treatment YSQ } \\
\text { Step } 2\end{array}$ & $.542^{\star}$ & 1.020 & .169 & .094 \\
\hline $\begin{array}{l}\text { Pre-treatment to mid-treatment YSQ } \\
\text { Step } 1\end{array}$ & .300 & .898 & .238 & .129 \\
\hline Pre-treatment to mid-treatment BSI & -.261 & .146 & & \\
\hline $\begin{array}{l}\text { Mid-treatment to end-of-treatment functional schema modes } \\
\text { Step } 2\end{array}$ & $-.627^{\star}$ & .568 & .299 & .235 \\
\hline $\begin{array}{l}\text { Pre-treatment to mid-treatment functional schema modes } \\
\text { Step } 1\end{array}$ & $-.418^{\star}$ & .620 & .449 & .370 \\
\hline Pre-treatment to mid-treatment BSI & -.254 & .194 & & \\
\hline $\begin{array}{l}\text { Mid-treatment to end-of-treatment dysfunctional schema modes } \\
\text { Step } 2\end{array}$ & $.573^{\star}$ & 1.004 & .294 & .230 \\
\hline $\begin{array}{l}\text { Pre-treatment to mid-treatment dysfunctional schema modes } \\
\text { Mid-to end-of-treatment YSQ } \\
\text { Step } 1\end{array}$ & .030 & .909 & .295 & .194 \\
\hline Pre-treatment to mid-treatment YSQ & -.338 & .164 & & \\
\hline $\begin{array}{l}\text { Mid-treatment to end-of-treatment BSI } \\
\text { Step } 2\end{array}$ & $.405^{\star}$ & .036 & .115 & .034 \\
\hline $\begin{array}{l}\text { Pre-treatment to mid-treatment BSI } \\
\text { Mid-to end-of-treatment functional schema modes } \\
\text { Step } 1\end{array}$ & $.622^{\star \star}$ & .034 & .430 & .349 \\
\hline Pre-treatment to mid-treatment functional schema modes & $-.400^{\star}$ & .187 & & \\
\hline $\begin{array}{l}\text { Mid-treatment to end-of-treatment BSI } \\
\text { Step } 2\end{array}$ & $-.644^{\star \star}$ & .050 & .374 & .317 \\
\hline $\begin{array}{l}\text { Pre-treatment to mid-treatment BSI } \\
\text { Mid-to end-of-treatment dysfunctional schema modes } \\
\text { Step } 1\end{array}$ & -.264 & .043 & .434 & .353 \\
\hline Pre-treatment to mid-treatment dysfunctional schema modes & -.277 & .161 & & \\
\hline $\begin{array}{l}\text { Mid-treatment to end-of-treatment BSI } \\
\text { Step } 2\end{array}$ & $.478^{\star}$ & .034 & .246 & .177 \\
\hline Pre-treatment to mid-treatment BSI & $.464^{\star}$ & .033 & .412 & .328 \\
\hline
\end{tabular}

Variables are residualised change scores; ${ }^{\star}$ significant at a 0.05 level (two-tailed); ${ }^{* *}$ significant at a 0.01 level (two-tailed).

\section{Further analyses: point-biserial correlation}

We further examined whether the outcome of treatment might be different in specific PDs. Therefore, we first specified the other specified personality disorders (OSPD) on the level of traits. We defined three dichotomous independent variables, borderline personality disorder (BPD) $(n=6)$, obsessive compulsive personality disorder/OSPD with obsessive compulsive traits $(n=7)$ and avoidant personality disorder/OSPD with avoidant traits $(n=10)$. As dependent variable the change score of BSI end-of-treatment to BSI pre-treatment was used. We found significant correlations and medium effect sizes between BSI change score and BPD $(r=-.44, p=.026)$ and between BSI change score and obsessive compulsive personality disorder/personality disorder with obsessive compulsive traits $(r=.48, p=.013)$. The negative correlation between BSI change score and BPD indicates that the patients diagnosed with BPD improved more on symptomatic distress at the end-of-treatment. The correlation between BSI change score and patients diagnosed with an obsessive compulsive disorder indicates an increase in symptomatic distress during treatment. The correlation between BSI change score and avoidant personality disorder/personality disorder with avoidant traits was not significant $(r=-.13, p=.54)$. 


\section{Discussion}

In this study we explored the applicability, feasibility and outcome of an adapted form of brief group schema therapy for older adults with mixed PDs. In summary, most patients showed a positive change in symptomatic distress, EMS, schema modes and personality functioning over time, but the magnitude of change differed from one patient to another. Of all patients, $4 \%$ recovered, $12 \%$ improved, $73 \%$ remained unchanged and $12 \%$ deteriorated. Comparing our results with the previous study by Videler et al. (2014) in 31 older out-patients with longstanding mood disorders and PD features, no significant changes on the outcome measures were found and we found smaller effect sizes for all process variables.

In retrospect, we probably changed too many aspects at the same time, as we aimed to improve the acceptability and effectiveness of schema therapy for older adults with PDs. Besides the agespecific adaptations to the treatment protocol, as described in the introduction, we also changed the nature of the population. Our current sample suffered from more severe pathology, as it consisted of a group of older adults with full PDs compared with the patients with PD features in the earlier study by Videler and colleagues (2014). The lower scores in symptomatic distress during pre- (mean 63.58 vs 74.29), mid- (mean 52.54 vs 70.60), and end-of-treatment (mean $48.00 v s$ 67.12) in the study by Videler et al. support this as a possible explanation.

Intermediate outcome analysis showed that the change in symptomatic distress at the beginning of the treatment predicted later positive change in EMS and dysfunctional schema modes. This was comparable to the results of Videler and colleagues (2014), who also found that changes in EMS co-occurred with changes in symptomatic distress.

In this study only three patients dropped out (10\%), compared with 11 patients in the study (26\%) by Videler et al. (2014). The low drop-out rate may suggest high acceptability by the patients, and also may suggest that the adaptations to the ST group protocol enhanced the acceptability for older adults.

We found a negative correlation between BSI change score and BPD, indicating that the patients diagnosed with BPD improved more at the end-of-treatment. The patients diagnosed with an obsessive compulsive disorder with obsessive compulsive traits showed significantly higher levels of symptomatic distress at the end of treatment than at the start of treatment. Cluster C PD patients extensively display emotional inhibited, over-controlling, avoiding and fearful behaviour. Emotional inhibition and avoidance can be longstanding, very rigid behavioural patterns with an adaptive value and a reinforcing effect, and are often very hard to break through in psychotherapy (Dimaggio et al., 2018). This again supports our hypothesis that in the current study patients showed more severe pathology than in the study by Videler et al. (2014). A randomized controlled trial (RCT) with patients with mainly cluster C PDs demonstrated successful treatment with group schema therapy (Bamelis et al., 2014), but treatment dosage and the duration of treatment were much higher than in the current study, that is 50 sessions over 36 months compared with 22 sessions in 6 months. Treatment dosage was also much higher in the individual ST study in older adults with cluster C PDs, with 50 sessions in 18 months (Videler et al., 2018).

Furthermore, preliminary results of an international RCT examining which format for delivery of schema therapy for BPD in younger adults produces the best outcome (format A: intensive group therapy only; or format B: a combination of group and individual therapy) support our hypothesis that a higher treatment dosage, and more specifically in a combination of group schema therapy and individual treatment sessions might yield better results in older adults as well (Arntz, 2019; Wetzelaer et al., 2014). In a RCT with patients with internalizing problems, Lorentzen et al. (2015) found that patients with PDs benefit from a more prolonged therapy compared with patients without a PD, who showed better results with a short group therapy. These findings are in line with the results from a previous meta-analysis who showed that 
long-term psychodynamic psychotherapy was superior to less intensive forms of psychotherapy in patients with PDs (Leichsenring and Rabung, 2011).

As older adults need more time to learn the schema language and to recognize the triggering of schemas and modes in their personal life, we lengthened the protocol with two pre-treatment sessions and two additional therapy sessions, but this may not have been enough, especially in patients with more severe personality pathology. Patients pointed out that they were more aware of their dysfunctional patterns; maybe they had not been able yet to work on behavioural change due to this ST treatment being too brief. In sum, older patients with PDs might benefit from more ST sessions, similar to the treatment dosage and treatment intensity in younger PD patients, ranging from 78 up to 300 sessions (Bamelis et al., 2014; Giesen-Bloo et al., 2006; Nordahl and Nysæter, 2005). They might also benefit most from a combination of group therapy and individual treatment sessions (Arntz, 2019).

Limitations of this study include a small sample size and the absence of a control condition, which limits the generalizability and restricts the number and types of analyses that could be carried out. More importantly, as mentioned above, in retrospect we adapted the protocol and also changed the inclusion criteria to older patients with a PD as main diagnosis. Moreover, compared with the study by Videler et al. (2014), in which all patients were recruited from a geriatric out-patient setting, in our study 10 patients were recruited from two tertiary care centres, both highly specialized in highly complex older PD patients. Accordingly, in retrospect, it is not possible to determine the extent to which our results can be ascribed to the more severe population or to the adaptions of the treatment protocol.

A final limitation is that we did not assess the acceptability of this adapted schema group therapy for older adults with psychotherapy process measures, nor with qualitative measures. In future feasibility studies of adaptions of schema therapy for older people, addition of qualitive research methods in a mixed method design might shed further light on whether adaptations enhance feasibility and treatment outcome in later life.

Despite these limitations, the present study adds to the emerging body of evidence for ST in older adults. It supports the notion that ST is a promising treatment for older adults with PDs and increases our knowledge on the development of adequate group ST protocols for older people. Both effectiveness studies focusing on the treatment of PDs as the main focus of therapy in older adults and the evidence for the effectiveness of group ST for other PDs than borderline PD are sparse (Penders et al., 2020; Skewes et al., 2015; van Alphen et al., 2015). Adding individual treatment sessions, and providing more therapy sessions, similar to the treatment dosages and intensity that have been found efficacious in younger BPD patients (Arntz, 2019), may lead to better treatment effects in older PD patients. This hypothesis may inform further research.

The question remains whether the modifications in the protocol will indeed enhance the efficacy of group schema therapy in older adults. This could be studied by randomly assigning older PD patients to the adapted treatment protocol or to a treatment as usual condition. Also, based on patient characteristics, it could be further investigated which dosage and intensity of treatment is needed. The effect of a higher treatment dosage and intensity might be investigated by comparing patients in the adapted treatment protocol with patients in a condition with a combination of group and individual ST. The effects of specific interventions for cluster B and cluster $\mathrm{C}$ personality disorders, like they were developed for younger adults (Baljé et al., 2016; Farrell et al., 2009), might be worth studying in older adults.

In sum, we set out to adapt brief group schema therapy for older adults to explore its feasability, acceptability and outcome, but learnt some important lessons for future research and clinical practice. First, we should not have changed both the protocol and included more complex PD patients. Second, when assessing acceptability, a mixed method design which includes qualitative methods is more appropriate. 
For clinical practice, this study inspired us to start with a more intensive group schema therapy treatment, that combines weekly group sessions with two-weekly individual sessions for a period of at least 12 months. Besides treating older adults as intensively as younger adults, group ST will most likely be more effective by providing the full array of group ST interventions, as is provided to younger adults. The original brief schema therapy protocol in the study by Videler et al. (2014) consisted of only CBT techniques. The experiential techniques of ST, like imagery and rescripting and chairwork, add extra power to the treatment (Arntz and Van Genderen, 2020) and have been found to contribute to ST in older adults in individual ST (Videler et al., 2018). The most promising age-specific adaptations to group ST are incorporating wisdom enhancement and reinforcing positive, or early adaptive, schemas. Whereas early maladaptive schema are formed in childhood, when basic emotional needs are not fulfilled, early adaptive schema (EAS) emerge during childhood, when core emotional needs are adequately met by primary caregivers (Lockwood and Perris, 2012). Integrating EAS may be important vehicles of change in schema therapy with older adults, because, as people age, and their EAS are no longer or not sufficiently triggered, EMS may become more prominent. This could also occur earlier in life but is more typical in ageing people. For a more extensive discussion of working with positive schemas in older adults, see Videler et al. (2020) and van Donzel et al. (2021). We are currently studying this more intensive group schema therapy protocol with a multiple baseline case series design to study the effects, combined with qualitative interviews to examine its feasibility.

\section{Key practice points}

(1) Group schema therapy for older people with PDs should be treated as intensively as younger people.

(2) When assessing acceptability of a new treatment, a mixed method design with qualitative methods is most appropriate.

(3) When studying a new treatment, do not change both the protocol and the patients included.

\section{Acknowledgements. None.}

Financial support. This research received no specific grant from any funding agency, commercial or not-for-profit sectors.

Conflicts of interest. The authors declare none.

Ethics statements. The study was approved by the Medical Ethic Committee of Zuyderland Hospital and the local research committees of the participating sites provided ethics approval. The trial was registered on 30 July 2017 in the Dutch Trial Register (NTR6563); the trial was conducted in the Netherlands between September 2017 and January 2020.

Data availability statement. The anonymised data are available to other researchers on reasonable request. The data are not publicly available due to their containing information that could compromise the privacy of research participants.

Author contributions. A.C. Videler: Investigation (equal), Methodology (lead), Project administration (lead), Supervision (lead), Writing-review \& editing (lead); K.A. van Beest: Formal analysis (equal), Investigation (equal), Project administration (supporting), Writing-original draft (equal); M.A. Ouwens: Formal analysis (equal), Writing-original draft (equal); G. Rossi: Formal analysis (lead), Writing-original draft (equal); R.J.J. van Royen: Conceptualization (equal), Investigation (equal), Writing-original draft (equal); S.P.J. van Alphen: Methodology (equal), Supervision (equal), Writing-original draft (equal), Writing-review \& editing (equal).

\section{Further reading}

Penders, K. A. P., Duimel-Peeters, I. G. P., Metsemakers, J. F. M., \& van Alphen, S. P. J. (2020). Personality disorders in older adults: a review of epidemiology, assessment and treatment. Current Psychiatry Reports, 22. https://doi.org/10.1007/ s11920-020-1133-x 
van Donzel, L., Ouwens, M.A., van Alphen, S.P.J., Bouwmeester, S., \& Videler, A.C. (2021). The effectiveness of adapted schema therapy for cluster $\mathrm{C}$ personality disorders in older adults: integrating positive schemas. Contemporary Clinical Trials Communications, 21, 100715. https://doi.org/10.1016/j.conctc.2021.100715

Videler, A. C., van Royen, R. J. J., Legra, M. J. H., \& Ouwens, M. A. (2020). Positive schemas in older adults: clinical implications and research suggestions. Behavioural and Cognitive Psychotherapy, 48, 481-491. https://doi.org/10.1017/ S1352465820000077

\section{References}

Arntz, A. (2019, September 20). An update of theory and research into schema therapy [Conference session]. Dutch schema therapy conference 2019, Utrecht, the Netherlands.

American Psychiatric Association (2013). Diagnostic and statistical manual of mental disorders (5th ed). https://doi.org/10. 1176/appi.books.9780890425596

Arntz, A., \& Van Genderen, H. (2020). Schema Therapy for Borderline Personality Disorder. John Wiley \& Sons.

Baljé, A., Greeven, A., van Giezen, A., Korrelboom, K., Arntz, A., \& Spinhoven, P. (2016). Group schema therapy versus group cognitive behavioral therapy for social anxiety disorder with comorbid avoidant personality disorder: study protocol for a randomized controlled trial. Trials, 17. https://doi.org/10.1186/s13063-016-1605-9

Bamelis, L. L. M., Evers, S. M. A. A., Spinhoven, P., \& Arntz, A. (2014). Results of a multicenter randomized controlled trial of the clinical effectiveness of schema therapy for personality disorders. American Journal of Psychiatry, 171, 305-322. https://doi.org/10.1176/appi.ajp.2013.12040518

Broersen, J., \& Van Vreeswijk, M. (2012). Schema therapy in groups: A short-term schema CBT protocol. In M. van Vreeswijk, M., J. Broersen, \& M. Nadort (Eds.). The Wiley-Blackwell handbook of schema therapy (pp. 373-381), John Wiley \& Sons.

Cockram, D. M., Drummond, P. D., \& Lee, C. W. (2010). Role and treatment of early maladaptive schemas in Vietnam veterans with PTSD. Clinical Psychology \& Psychotherapy, 17, 165-182. https://doi.org/10.1002/cpp.690

Cohen, J. (1988). The effect size index: d. Statistical Power Analysis for the Behavioral Sciences, 2, 284-288.

De Beurs, E. (2011). Brief Symptom Inventory: Dutch Manual. PITS.

Debast, I., Rossi, G., \& van Alphen, S. P. J. (2018). Age-neutrality of a brief assessment of the section III alternative model for personality disorders in older adults. Assessment, 25, 310-323.

Derogatis, L. R. (1975a). The Brief Symptom Inventory. Clinical Psychometric Research.

Derogatis, L. R. (1975b). The Symptom Checklist-90-R. Clinical Psychometric Research.

Dimaggio, G., MacBeth, A., Popolo, R., Salvatore, G., Perrini, F., Raouna, A., Osam, C. S., Buonocore, L., Bandiera, A., \& Montano, A. (2018). The problem of overcontrol. Perfectionism, emotional inhibition, and personality disorders. Comprehensive Psychiatry, 83, 71-78. https://doi.org/10.1016/j.comppsych.2018.03.005

Farrell, J. M., Shaw, I. A., \& Webber, M. A. (2009). A schema-focused approach to group psychotherapy for outpatients with borderline personality disorder: a randomized controlled trial. Journal of Behavior Therapy and Experimental Psychiatry, 40, 317-328. https://doi.org/10.1016/j.jbtep.2009.01.002

Finkel, S. E. (1995). Causal Analysis with Panel Data. Sage.

First, M. B., Gibbon, M., Spitzer, R. L., Benjamin, L. S., \& Williams, J. B. (1997). Structured Clinical Interview for DSM-IV Axis II Personality Disorders: SCID-II. American Psychiatric Publications.

Giesen-Bloo, J., Van Dyck, R., Spinhoven, P., Van Tilburg, W., Dirksen, C., Van Asselt, T., Kremers, I., Nadort, M., \& Arntz, A. (2006). Outpatient psychotherapy for borderline personality disorder: randomized trial of schema-focused therapy vs transference-focused psychotherapy. Archives of General Psychiatry, 63, 649-658. https://doi:10.1001/ archpsyc.63.6.649

Hutsebaut, J., Videler, A. C., Verheul, R., \& van Alphen, S. P. J. (2019). Managing borderline personality disorder from a life course perspective: clinical staging and health management. Personality Disorders: Theory, Research, and Treatment, 10, 309-316. https://doi.org/10.1037/per0000341

Lambert, M. J., Hansen, N. B., \& Bauer, S. (2008). Assessing the clinical significance of outcome results. In A. Nezu, \& C. M. Nezu (Eds.) Evidence-Based outcome research: A practical guide to conducting randomized controlled trials for psychosocial interventions (pp. 359-378). Oxford University Press.

Leichsenring, F., \& Rabung, S. (2011). Long-term psychodynamic psychotherapy in complex mental disorders: update of a meta-analysis. British Journal of Psychiatry, 199, 15-22. https://doi.org/10.1192/bjp.bp.110.082776

Lobbestael, J., Leurgans, M., \& Arntz, A. (2011). Inter-rater reliability of the structured clinical interview for DSM-IV axis I disorders (SCID I) and axis II disorders (SCID II). Clinical Psychology \& Psychotherapy, 18, 75-79. https://doi.org/10. $1002 /$ cpp.693

Lobbestael, J., van Vreeswijk, M. F., Spinhoven, P., Schouten, E., \& Arntz, A. (2010). Reliability and validity of the short Schema Mode Inventory (SMI). Behavioural and Cognitive Psychotherapy, 38, 437-458. https://doi.org/10.1017/ S1352465810000226 
Lobbestael, J., Van Vreeswijk, M. F., Arntz, A., Spinhoven, P., \& 't Hoen, T. (2005). Dutch Translation of the Young Atkinson Mode Inventory (YAMI). Maastricht, The Netherlands: Maastricht University.

Lockwood, G., \& Perris, P. (2012). A new look at core emotional needs. In van Vreeswijk, M., Broersen, J., \& Nadort, M. (eds), The Wiley-Blackwell Handbook of ST: Theory, Research and Science (pp. 41-66). Wiley-Blackwell.

Lorentzen, S., Ruud, T., Fjeldstad, A., \& Høglend, P. A. (2015). Personality disorder moderates outcome in short-and longterm group analytic psychotherapy: a randomized clinical trial. British Journal of Clinical Psychology, 54, 129-146. https:// doi.org/10.1111/bjc.12065

Malogiannis, I. A., Arntz, A., Spyropoulou, A., Tsartsara, E., Aggeli, A., Karveli, S., Vlavianou, M., Pehilvanidis, A., Papadimitriou, G. N., \& Zervas, I. (2014). Schema therapy for patients with chronic depression: a single case series study. Journal of Behavior Therapy and Experimental Psychiatry, 45, 319-329. https://doi-org.tilburguniversity.idm.oclc. org/10.1016/j.jbtep.2014.02.003

Nadort, M., Arntz, A., Smit, J. H., Giesen-Bloo, J., Eikelenboom, M., Spinhoven, P., Van Asselt, T., Wensing, M., \& van Dyck, R. (2009). Implementation of outpatient schema therapy for borderline personality disorder with versus without crisis support by the therapist outside office hours: a randomized trial. Behaviour Research and Therapy, 47, 961-973. https://doi.org/10.1016/j.brat.2009.07.013

Newton-Howes, G., Clark, L. A., \& Chanen, A. (2015). Personality disorder across the life course. The Lancet, 385, 727-734. https://doi.org/10.1016/S0140-6736(14)61283-6

Nordahl, H. M., \& Nysæter, T. E. (2005). Schema therapy for patients with borderline personality disorder: a single case series. Journal of Behavior Therapy and Experimental Psychiatry, 36, 254-264. https://doi.org/10.1016/j.jbtep.2005.05.007

Pauwels, E., Claes, L., Dierckx, E., Debast, I., van Alphen, S. P. J., Rossi, G., Schotte, C., Santens, E., \& Peuskens, H. (2014). Age neutrality of the Young Schema Questionnaire in patients with a substance use disorder. International Psychogeriatrics, 26, 1317-1326. https://doi.org/10.1017/S1041610214000519

Penders, K. A. P., Duimel-Peeters, I. G. P., Metsemakers, J. F. M., \& van Alphen, S. P. J. (2020). Personality disorders in older adults: a review of epidemiology, assessment and treatment. Current Psychiatry Reports, 22. https://doi.org/10.1007/ s11920-020-1133-x

Renner, F., Arntz, A., Peeters, F. P., Lobbestael, J., \& Huibers, M. J. (2016). Schema therapy for chronic depression: results of a multiple single case series. Journal of Behavior Therapy and Experimental Psychiatry, 51, 66-73. https://doi-org. tilburguniversity.idm.oclc.org/10.1016/j.jbtep.2015.12.001

Renner, F., van Goor, M., Huibers, M., Arntz, A., Butz, B., \& Bernstein, D. (2013). Short-term group schema cognitivebehavioral therapy for young adults with personality disorders and personality disorder features: associations with changes in symptomatic distress, schemas, schema modes and coping styles. Behaviour Research and Therapy, 51, 487-492. https:// doi.org/10.1016/j.brat.2013.05.011

Rijkeboer, M. M., \& van den Bergh, H. (2006). Multiple group confirmatory factor analysis of the Young SchemaQuestionnaire in a Dutch clinical versus non-clinical population. Cognitive therapy and research, 30(3), 263-278. https://doi.org/10.1007/s10608-006-9051-8

Rijkeboer, M. M., Van den Bergh, H., \& Van den Bout, J.. (2005). Stability and discriminative power of the Young Schema Questionnaire. Journal of Behavioral Therapy and Experimental Psychiatry, 36, 129-144. doi: 10.1016/j.jbtep.2004.08.005

Ro, E., \& Clark, L. A. (2009). Psychosocial functioning in the context of diagnosis: assessment and theoretical issues. Psychological Assessment, 21, 313. https://psycnet.apa.org/doi/10.1037/a0016611

Rosnow, R. L., \& Rosenthal, R. (1996). Computing contrasts, effect sizes, and counternulls on other people's published data: general procedures for research consumers. Psychological Methods, 1, 331.

Rosowsky, E., Young, A. S., Malloy, M. C., van Alphen, S. P. J, \& Ellison, J. M. (2018). A cross-validation Delphi method approach to the diagnosis and treatment of personality disorders in older adults. Aging \& Mental Health, 22, 371-378. https://doi.org/10.1080/13607863.2016.1261796

Rossi, G., Debast, I., \& van Alphen, S. P. J. (2016). Measuring personality functioning in older adults: construct validity of the Severity Indices of Personality Functioning-Short Form (SIPP-SF). Aging \& Mental Health, 21, 703-711. https://doi.org/10. 1080/13607863.2016.1154012

Simpson, S. G., Morrow, E., \& Reid, C. (2010). Group schema therapy for eating disorders: a pilot study. Frontiers in Psychology, 1, 182. https://doi.org/10.3389/fpsyg.2010.00182

Skewes, S. A., Samson, R. A., Simpson, S. G., \& van Vreeswijk, M. F. (2015). Short-term group schema therapy for mixed personality disorders: a pilot study. Frontiers in Psychology, 5, 1592. https://doi.org/10.3389/fpsyg.2014.01592

Sterk, F., \& Rijkeboer, M. M. (1997). Schema-vragenlijst [schema-questionnaire]. Ambulatorium Utrecht University.

Thiel, N., Jacob, G. A., Tuschen-Caffier, B., Herbst, N., Külz, A. K., Hertenstein, E., Nissen, C., \& Voderholzer, U. (2016). Schema therapy augmented exposure and response prevention in patients with obsessive-compulsive disorder: feasibility and efficacy of a pilot study. Journal of Behavior Therapy and Experimental Psychiatry, 52, 59-67. https://doi-org. tilburguniversity.idm.oclc.org/10.1016/j.jbtep.2016.03.006

van Alphen, S. P. J., Bolwerk, N., Videler, A. C., Tummers, J. H. A., Van Royen, R. J. J., Barendse, H. P. J., Verheul, R., \& Rosowsky, E. (2012). Age related aspects and clinical implementations of diagnosis and treatment of personality disorders in older adults. Clinical Gerontologist, 35, 27-41. https://doi.org/10.1080/07317115.2011.628368 
van Alphen, S. P. J., Engelen, G. J. J. A., Kuin, Y., Hoijtink, H. J. A., \& Derksen, J. J. L. (2006). A preliminary study of the diagnostic accuracy of the Gerontological Personality Disorders Scale (GPS). International Journal of Geriatric Psychiatry, 21, 862-868. https://doi.org/10.1002/gps.1572

van Alphen, S. P. J., Van Dijk, S. D. M., Videler, A. C., Rossi, G., Dierckx, E., Bouckaert, F., \& Voshaar, R. O. (2015). Personality disorders in older adults: emerging research issues. Current Psychiatry Reports, 17, 538. https://doi.org/10.1007/ s11920-014-0538-9

van Donzel, L., Ouwens, M.A., van Alphen, S.P.J., Bouwmeester, S., \& Videler, A.C. (2021). The effectiveness of adapted schema therapy for cluster $\mathrm{C}$ personality disorders in older adults: integrating positive schemas. Contemporary Clinical Trials Communications, 21, 100715. https://doi.org/10.1016/j.conctc.2021.100715

van Reijswoud, B., Debast, I., Videler, A. C., Rossi, G., Lobbestael, J., Segal, D. L., \& van Alphen, S. P. J. (2021). Severity Indices of Personality Problems - Short Form (SIPP-SF) in old age psychiatry: reliability and validity. Journal of Personality Assessment, 21, 703-711. https://doi.org/10.1080/00223891.2020.1743710

van Vreeswijk, M. F., Spinhoven, P., Eurelings-Bontekoe, E. H. M., \& Broersen, J. (2014). Changes in symptom severity, schemas and modes in heterogeneous psychiatric patient groups following short-term schema cognitive-behavioural group therapy: a naturalistic pre-treatment and post-treatment design in an outpatient clinic. Clinical Psychology \& Psychotherapy, 21, 29-38. https://doi.org/10.1002/cpp.1813

Verheul, R., Andrea, H., Berghout, C. C., Dolan, C., Busschbach, J. J., Kroft, P. J. V. D., Bateman, A.W., \& Fonagy, P. (2008). Severity Indices of Personality Problems (SIPP-118): development, factor structure, reliability, and validity. Psychological Assessment, 20, 23-34. https://psycnet.apa.org/doi/10.1037/1040-3590.20.1.23

Videler, A. C., Rossi, G., Schoevaars, M., Van der Feltz-Cornelis, C. M., \& van Alphen, S. P. J. (2014). Effects of schema group therapy in older outpatients: a proof of concept study. International Psychogeriatrics, 26, 1709-1717. https://doi.org/ 10.1017/S1041610214001264

Videler, A. C., van Alphen, S. P. J., Van Royen, R. J. J., Van der Feltz-Cornelis, C. M., Rossi, G., \& Arntz, A. (2018). Schema therapy for personality disorders in older adults: a multiple-baseline study. Aging and Mental Health, 22, 738-747. https://doi.org/10.1080/13607863.2017.1318260

Videler, A. C., Van der Feltz-Cornelis, C. M., Rossi, G., Van Royen, R. J. J., Rosowsky, E., \& van Alphen, S. P. J. (2015). Psychotherapeutic treatment levels of personality disorders in older adults. Clinical Gerontologist, 38, 325-341. https://doi. org/10.1080/07317115.2015.1032464

Videler, A. C., van Royen, R. J. J., Legra, M. J. H., \& Ouwens, M. A. (2020). Positive schemas in older adults: clinical implications and research suggestions. Behavioural and Cognitive Psychotherapy, 48, 481-491. https://doi.org/10.1017/ S1352465820000077

Videler, A. C., van Royen, R. J. J., van Alphen, S. P. J, Rossi, G. \& van der Feltz-Cornelis, C. M. (2017). Adapting schema therapy for personality disorders in older adults. International Journal of Cognitive Therapy, 10, 62-78. https://doi.org/10. 1521/ijct.2017.10.1.62

Weertman, A., Arntz, A., \& Kerkhofs, M. L. M. (2000). Gestructureerd diagnostisch interview voor DSM-IV persoonlijkheidsstoornissen (SCID II) [Structural and Clinical Interview for DSM-IV personality disorders (SCID II)]. Swets Test Publisher.

Wetzelaer, P., Farrell, J., Evers, S. M., Jacob, G. A., Lee, C. W., Brand, O., van Breukelen, G., Fassbindder, E., Fretwell, H., Harper, R.P., Lavender, A., Lockwood, G., Malogiannis, I. A., Schweiger, U., Startup, H., Stevenson, T., Zarbock, G., \& Arntz, A. (2014). Design of an international multicentre RCT on group schema therapy for borderline personality disorder. BMC Psychiatry, 14, 1-15. https://doi.org/10.1186/s12888-014-0319-3

Young, J. E. (1990). Cognitive Therapy for Personality Disorders: A Schema-Focused Approach. Professional Resource Press.

Young, J. E., \& Brown, G. (1994). Young Schema-Questionnaire (2nd edn). In Young, J. E. (ed), Cognitive Therapy for Personality Disorders: A Schema-Focused Approach. Professional Resource Press.

Young, J. E., Klosko, J. S., \& Weishaar, M. E. (2003). Schema Therapy: A Practitioner's Guide. Guilford Press.

Cite this article: Videler AC, van Beest KA, Ouwens MA, Rossi G, van Royen RJJ, and van Alphen SPJ. Adapting group schema therapy for older adults with personality disorders: lessons learnt. The Cognitive Behaviour Therapist. https:// doi.org/10.1017/S1754470X21000325 